\title{
X51 - Biomedical Applications of microCT in Hard and Soft Tissues - Going Beyond the Bone.
}

Keiko Takahashi ${ }^{5}$, Mohamed A.A. Saleh ${ }^{6}$, Nicole Fleming ${ }^{3,4}$, Takamune Takahashi ${ }^{5}$, and Daniel S. Perrien ${ }^{1,2,3,4}$

1. Department of Veteran's Affairs, Tennessee Valley Healthcare System, Nashville TN, USA; ${ }^{2}$ Department of Orthopaedic Surgery and Rehabilitation, ${ }^{3}$ Vanderbilt University Institute of Imaging Science, and ${ }^{4}$ Vanderbilt Center for Bone Biology, ${ }^{5}$ Department of Nephrology, ${ }^{6}$ Department of Medicine, Vanderbilt University, Nashville TN, USA.

Although x-ray micro computed tomography $(\mu \mathrm{CT})$ originally gained widespread popularity in the skeletal field, new techniques have proven its utility in many applications. MicroCT-based analyses are common in skeletal literature, but investigators must carefully match their approach to the biological hypothesis. Likewise, $\mu$ CT-based analyses of soft tissues and vascular structure require consideration of several factors to insure that the data produced addresses the proper biological question. Hence, this tutorial will review the advantages and limitations of $\mu \mathrm{CT}$ using established and novel applications to illustrate the factors that must be considered to design appropriate acquisition and analytical protocols.

MicroCT systems fall into two broad categories: synchrotron, which uses a monochromatic x-ray beam, or the much more common and affordable polychromatic instruments. The combination of micro-focal spot generators, cone-beam, geometry, precise specimen manipulation, and high pixel density detectors allows state-of-the-art polychromatic systems to achieve isotropic voxel sizes of less than 1/8000th of the field of view and as small as $10 \mathrm{~nm}$. However, true resolution of $\mu \mathrm{CT}$ imaging is more dependent on signal-to-noise ratio and background contrast. Hence, successful imaging at extremely high resolutions depends on optimization of several factors that often have opposing consequences.

Regardless of the tissue or material analyzed, $\mu \mathrm{CT}$ data is only as good as the acquisition protocol, precision of the volume of interest, and the appropriateness of the VOI to biological hypothesis. Hence, great care and consideration must be given to first identifying reproducible anatomic reference points and then defining a volume of interest relative to the reference point that will yield data relevant to the proper biological question.

The three-dimensional and nondestructive nature of $\mu \mathrm{CT}$ imaging makes it very appealing as a primary or secondary endpoint for studies aiming to assess structural features of small specimens. However, one must be able to secure the specimen of interest in a gas or solution with substantially different x-ray attenuation than the material of interest without damaging or deforming the specimen. This can be especially challenging for those interested in soft tissues or other low attenuation materials that must remain hydrated during image acquisition.

To address this issue, many techniques have been developed to "stain" ex vivo or synthetic specimens with contrast agents. For example, the ionic iodine-based contrast Hexabrix, is particularly useful to visualize cartilage and segment it from both bone and surrounding soft tissues due to the cationic nature of proteoglycans in the cartilage matrix [1] (Figure 1). Others have used osmium tetraoxide, which differentially "stains" numerous soft organs, and is particularly useful in ex vivo whole body imaging [2]. However, vascular imaging and analysis, using either lead- or barium-based contrast agents [3], 
may be the most common and popular biological application of $\mu \mathrm{CT}$ outside of mineralized tissues. The importance of vascular structure can only be fully understood in a volumetric analysis, and $\mu \mathrm{CT}$ analysis of contrast-enhanced vascular casts appears to be the only method to address these questions with reasonable through-put and cost.

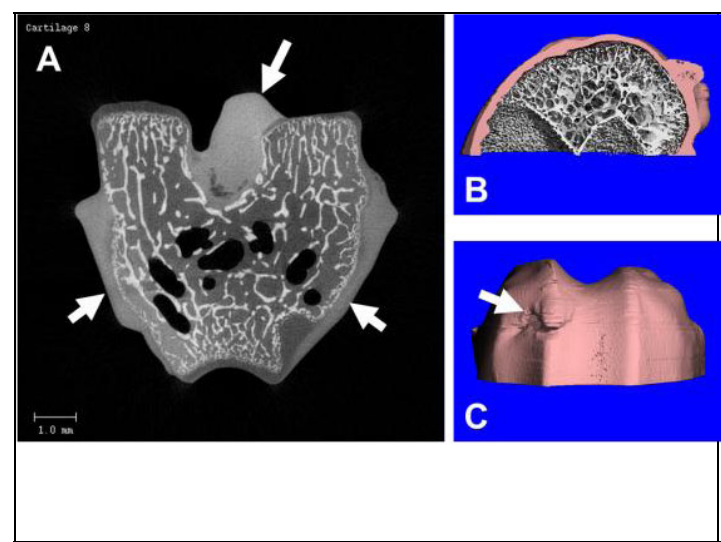

Figure 1 - Clinical contrast agents such as Hexabrix, can be used to analyze cartilage by ex vivo microCT. (A) As shown in the cross sectional image of a rat distal femur, soaking specimens in diluted contrast agent imparts cartillagenous elements (arrows) with a radiopacity between that of bone and the surrounding air. (B and $\mathrm{C}$ ) The cartilage (pink) can easily be segmented from the underlying bone (grey) for three dimensional image reconstruction and analysis. In addition to thickness, volume, and surface area of intact cartilage, defects $(\mathrm{C}$, arrow) are also easily visualized and quantified.

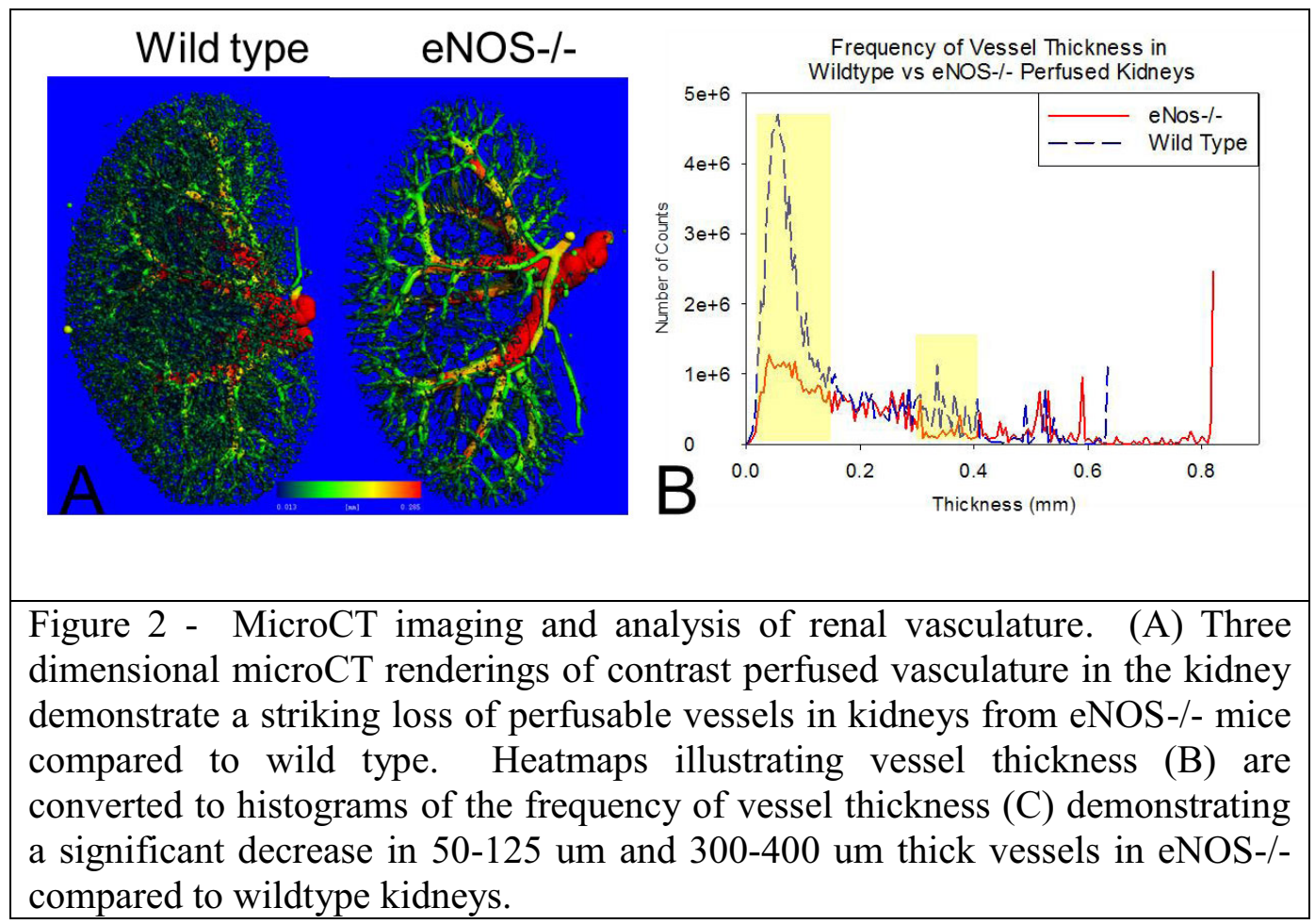

Literature Cited

1. Xie, L., et al., Quantitative assessment of articular cartilage morphology via EPIC-microCT. Osteoarthritis Cartilage, 2009. 17(3): p. 313-20.

2. Johnson, J.T., et al., Virtual histology of transgenic mouse embryos for high-throughput phenotyping. PLoS Genet, 2006. 2(4): p. e61.

3. Roche, B., et al., Structure and quantification of microvascularisation within mouse long bones: what and how should we measure? Bone, 2012. 50(1): p. 390-9. 